\title{
Effects of metformin on the glycemic control, lipid profile, and arterial blood pressure of type 2 diabetic patients with metabolic syndrome already on insulin
}

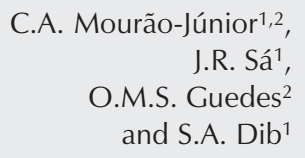

C.A. Mourão-Júnior ${ }^{1,2}$,

J.R. Sá1,

O.M.S. Guedes ${ }^{2}$ and S.A. Dib ${ }^{1}$

\author{
'Disciplina de Endocrinologia, Universidade Federal de São Paulo, \\ São Paulo, SP, Brasil \\ ${ }^{2}$ Departamento de Fisiologia, Universidade Federal de Juiz de Fora, \\ Juiz de Fora, MG, Brasil
}

\section{Correspondence}

C.A. Mourão Júnior

Rua General Mário Xavier, 64

36033-170 Juiz de Fora, MG

Brasil

Fax: +55-32-3215-3130

E-mail: mouraojr@terra.com.br

Publication supported by FAPESP.

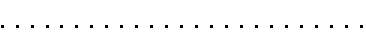

Received September 12, 2005

Accepted February 13, 2006

\begin{abstract}
Fifty-seven type 2 diabetic patients with metabolic syndrome and on insulin were assessed by a paired analysis before and 6 months after addition of metformin as combination therapy to evaluate the impact of the association on glycemic control, blood pressure, and lipid profile. This was a historical cohort study in which the files of type 2 diabetic patients with metabolic syndrome on insulin were reviewed. The body mass index (BMI), waist circumference, lipid profile, A1C level, fasting blood glucose level, daily dose of NPH insulin, systolic blood pressure, and diastolic blood pressure were assessed in each patient before the start of metformin and 6 months after the initiation of combination therapy. Glycemic control significantly improved $(\mathrm{P}<$ $0.001)$ after the addition of metformin $(1404.4 \pm 565.5 \mathrm{mg} /$ day $)$, with $14 \%$ of the 57 patients reaching A1C levels up to $7 \%$, and $53 \%$ reaching values up to $8 \%$. There was a statistically significant reduction $(\mathrm{P}<0.05)$ of total cholesterol $(229.0 \pm 29.5$ to $214.2 \pm 25.0 \mathrm{mg} /$ $\mathrm{dL}), \mathrm{BMI}\left(30.7 \pm 5.4\right.$ to $\left.29.0 \pm 4.0 \mathrm{~kg} / \mathrm{m}^{2}\right)$, waist circumference $(124.6$ \pm 11.7 to $117.3 \pm 9.3 \mathrm{~cm}$ ), and daily necessity of insulin. The reduction of total cholesterol occurred independently of the reductions of A1C $(9.65 \pm 1.03$ to $8.18 \pm 1.01 \%)$ and $\mathrm{BMI}$ and the reduction of BMI and WC did not interfere with the improvement of A1C. In conclusion, our study showed the efficacy of the administration of metformin and insulin simultaneously without negative effects. No changes were detected in HDL-cholesterol or blood pressure.
\end{abstract}

\section{Introduction}

The need for strict glycemic control in order to avoid or postpone the development of late complications in patients with type 2 diabetes mellitus (DM2) has been well established (1). It has been shown that the
Key words

- Type 2 diabetes mellitus

- Metabolic syndrome

- Metformin

- Blood pressure

- Cholesterol

- Triglycerides onset of complications may be related to glycosylated hemoglobin (A1C) levels higher than $7 \%$, a situation that is even more likely if glycemic control is poor, with A1C levels above $8 \%$ (2). Although the initial management of DM2 involves lifestyle changes and oral hypoglycemic drugs, a significant num- 
ber of patients will need insulin in order to reach satisfactory glycemic control (3). Furthermore, DM2 does not mean glycemic alterations alone since this disease is associated with cardiovascular risk factors such as dyslipidemia, systemic arterial hypertension, and obesity (4). This association is clinically relevant since over $50 \%$ of deaths among DM2 patients are due to cardiovascular disease (5).

Treatment of DM2 must include pharmacological agents able to improve not only glycemic levels, but also blood pressure (BP), lipid levels, and body weight, since in approximately $90 \%$ of type 2 diabetics hyperglycemia is associated with other cardiovascular factors that constitute the metabolic syndrome.

Several studies $(6,7)$ have demonstrated that, in order to prevent the development and progression of chronic complications of diabetes, a comprehensive approach to all elements of the metabolic syndrome is required. The main feature of the metabolic syndrome, which can be clinically assessed by measurement of the waist circumference (WC) whenever more sophisticated laboratory methods are not available (8), is insulin resistance.

After metformin proved to be effective in reducing insulin resistance (9), several studies were undertaken to assess its effects on total cholesterol (TC), triglycerides (TG), and HDL-cholesterol (HDL-C) levels, and also on BP and body mass index (BMI). However, there is no consensus about its beneficial effects on these parameters (10). Most studies have analyzed independent samples, comparing patients on insulin with patients on combined insulin-metformin therapy (11-13) with no conclusive results.

The objective of the present study was to assess a group of DM2 patients with metabolic syndrome, on insulin, before and 6 months after the introduction of metformin as combined therapy, so as to evaluate the effect of this association on glycemic control, BP, and the lipid profile.

\section{Patients, Material and Methods}

\section{Data collection}

We undertook a historical cohort study by assessing the medical files of type 2 diabetic patients with metabolic syndrome, on insulin, seen at a public diabetes-dedicated facility in a medium-sized Brazilian city (Juiz de Fora). All patients were followed up bimonthly by a team composed of four endocrinologists, a nutritionist, and a diabetes-trained nurse.

\section{Patient selection}

We selected files of patients who had been on twice daily injections of NPH insulin and who had metformin added to their therapy. Any file that showed any dietary indiscretion or drug misuse was not utilized in the study. A dietary indiscretion was defined as the intake of sucrose-containing foods three or more times a week, not considering the use of sweet foods taken during hypoglycemic episodes. A drug misuse was defined as any failure to take the prescribed drugs, as reported by the patient.

To avoid the inclusion of late-onset type $1 \mathrm{DM}$ patients in the sample, we studied only patients with documented control of their disease with oral hypoglycemic drugs alone for at least 5 years before they began insulin treatment, and who showed no evidence of previous episodes of ketoacidosis. All patients followed the same diet, prescribed and monitored by the nutritionist.

The patients were diagnosed as having metabolic syndrome if they fulfilled at least 3 of the National Cholesterol Education Program - Adult Treatment Panel III (NCEP ATP III) criteria (14). Although other criteria exist, as the World Health Organization criteria, the NCEP ATP III was the most widely used classification at present (6).

All patients had type 2 diabetes mellitus (blood glucose $>126 \mathrm{mg} / \mathrm{dL}$ ), high triglycer- 
ide levels ( $>150 \mathrm{mg} / \mathrm{dL})$, and increased WC (women: $>88 \mathrm{~cm}$; men: $>102 \mathrm{~cm}$ ). Table 1 shows the patients' characteristics.

A patient on anti-hypertensive or lipidlowering drugs was only included if there was no change in the dosages of these drugs throughout the study period. Full attendance of scheduled consultations was another inclusion criterion.

\section{Variables studied}

BMI, WC, lipid profile (TC, HDL-C, and $\mathrm{TG})$, A1C, fasting blood glucose (FBG), daily dose of NPH insulin, systolic blood pressure (SBP), and diastolic blood pressure (DBP) were measured in each patient before the start of metformin and 6 months after initiation of combined therapy.

Weight measurements were made only once, with a digital scale with a maximum capacity of $180 \mathrm{~kg}$ and precision of up to 100 $\mathrm{g}$. The patients were weighed in their underwear after carefully stepping onto the center of the scale.

Height was measured only once, with a 1-mm precision tape measure fixed to the wall, with the zero mark at floor level. The patients were standing, with bare feet kept together, and with heels and occiput touching the tape.

For WC measurement, the patients were standing, with abdomen relaxed, upper limbs falling along the body, and the tape placed horizontally, halfway between the lower margin of the last rib and the iliac crest; the measurements were made with the tape firmly applied to the pelvis but without tissue compression. A flexible 1-mm precision tape measure was used.

Blood pressure was measured three times within a 10-min interval on the left arm, with the patient in the supine position, after a 5min rest, with a calibrated mercury sphygmomanometer.

TC, HDL-C, TG, and FBG levels were measured by the colorimetric method, with maximum acceptable values of $240 \mathrm{mg} / \mathrm{dL}$ (TC), $200 \mathrm{mg} / \mathrm{dL}$ (TG), and $110 \mathrm{mg} / \mathrm{dL}$ (FBG). The minimum acceptable value for HDL-C was $35 \mathrm{mg} / \mathrm{dL}$. A1C was measured by high-performance liquid chromatography (normal reference for stable A1C in non-diabetic patients is 2.8 to $5.2 \%$, according to the National Glycohemoglobin Standardization Program). All laboratory determinations were performed automatically. The study was approved by the local Ethics Committee and all patients signed written informed consent.

\section{Statistical analysis}

The data are reported as means $\pm \mathrm{SD}$, percentages $(\%)$ or $95 \%$ confidence intervals $(95 \% \mathrm{CI})$. All tests were two-tailed, and the level of significance was set at $\mathrm{P}<5 \%$.

In order to compare the means of the study variables before and after the introduction of metformin, a paired $t$-test with $95 \% \mathrm{CI}$ of the means was used. If the $t$-test showed a difference for any variable studied, Pearson's correlation test was performed to check for possible intervening variables (co-variance) by the analysis of the coefficient of determination $\left(\mathrm{r}^{2}\right)$ and $\mathrm{r}$ 95\% CI. All assumptions for the undertaking of parametric tests were checked and accounted for. The statistical package GraphPad InStat, version 3.06, for Windows (San Diego, CA, USA) was used in the analyses.

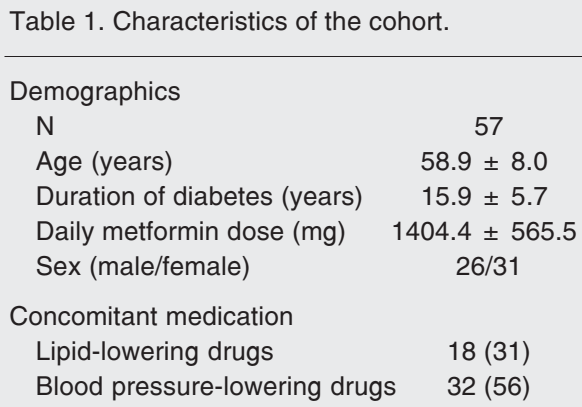

Data are reported as means \pm SD or $\mathrm{N}(\%)$. 


\section{Results}

Fifty-seven files met the inclusion criteria and were analyzed in this study.

There was a statistically significant reduction of the daily dose of NPH insulin, of BMI, WC and TC, and TG levels when the values before and 6 months after metformin were compared. Neither BP (SBP or DBP), nor HDL-C suffered any alteration. Table 2 summarizes the comparative measurements.

Glycemic control improved significantly $(\mathrm{P}<0.0001)$ after the introduction of metformin, with a reduction of mean FBG and A1C levels (Table 2). Furthermore, $14 \%$ of the patients reached A1C levels of up to $7 \%$,

Table 2. Comparison of clinical and laboratory variables before and 6 months after the introduction of metformin.

\begin{tabular}{lccc}
\hline & Before & After & $95 \% \mathrm{Cl}$ \\
\hline A1C $(\%)$ & $9.65 \pm 1.03$ & $8.18 \pm 1.01^{*}$ & 1.09 to 1.86 \\
FBG $(\mathrm{mg} / \mathrm{dL})$ & $215.3 \pm 28.0$ & $167.2 \pm 27.3^{*}$ & 37.5 to 58.6 \\
Daily insulin dose $\left(\mathrm{IU} \mathrm{kg}{ }^{-1}\right.$ day $\left.^{-1}\right)$ & $0.83 \pm 0.39$ & $0.69 \pm 0.36^{*}$ & 0.02 to 0.26 \\
BMI $\left(\mathrm{kg} / \mathrm{m}^{2}\right)$ & $30.7 \pm 5.4$ & $29.0 \pm 4.0^{*}$ & 0.1 to 3.3 \\
Waist circumference $(\mathrm{cm})$ & $124.6 \pm 11.7$ & $117.3 \pm 9.3^{*}$ & 3.6 to 10.9 \\
Total cholesterol $(\mathrm{mg} / \mathrm{dL})$ & $229.0 \pm 29.5$ & $214.2 \pm 25.0^{*}$ & 4.9 to 24.7 \\
HDL cholesterol $(\mathrm{mg} / \mathrm{dL})$ & $37.5 \pm 8.4$ & $38.0 \pm 6.3$ & -3.4 to 2.3 \\
Triglycerides $(\mathrm{mg} / \mathrm{dL})$ & $236.2 \pm 40.2$ & $218.5 \pm 42.4^{*}$ & 1.5 to 34.0 \\
Systolic blood pressure $(\mathrm{mmHg})$ & $158 \pm 25$ & $156 \pm 18$ & -7 to 11 \\
Diastolic blood pressure $(\mathrm{mmHg})$ & $92 \pm 11$ & $90 \pm 14$ & -3 to 6 \\
\hline
\end{tabular}

Data are reported as means $\pm \mathrm{SD}$. $\mathrm{A} 1 \mathrm{C}=$ glycohemoglobin level; $\mathrm{FBG}=$ fasting blood glucose. ${ }^{*} \mathrm{P}<0.05$ compared to before metformin treatment (Student $t$-test).

Table 3. Pearson's correlation test applied to pairs of variables.

\begin{tabular}{lcccc}
\hline Variables & P value & $r$ & $95 \% \mathrm{Cl}$ & $r^{2}$ \\
\hline TC and A1C & 0.597 & -0.072 & -0.326 to 0.193 & $0.5 \%$ \\
TC and BMI & 0.237 & 0.159 & -0.106 to 0.403 & $2.5 \%$ \\
TC and WC & 0.555 & 0.079 & -0.185 to 0.334 & $0.6 \%$ \\
TG and A1C & 0.605 & 0.070 & -0.194 to 0.325 & $0.5 \%$ \\
TG and BMI & 0.007 & 0.354 & 0.102 to 0.562 & $12.5 \%$ \\
TG and WC & 0.033 & 0.282 & 0.023 to 0.505 & $8 \%$ \\
A1C and BMI & 0.056 & 0.254 & -0.007 to 0.483 & $6.4 \%$ \\
A1C and WC & 0.926 & 0.012 & -0.272 to 0.249 & $0.01 \%$ \\
\hline
\end{tabular}

$\mathrm{TC}=$ total cholesterol; $\mathrm{A} 1 \mathrm{C}=$ glycohemoglobin level; $\mathrm{BMI}=$ body mass index; $\mathrm{WC}=$ waist circumference; TG = triglycerides; $r=$ correlation coefficient; $r^{2}=$ coefficient of determination. ${ }^{*} \mathrm{P}<0.05$.
$53 \%$ had A1C values of up to $8 \%$, and $47 \%$ had unsatisfactory glycemic control. Before metformin introduction, all patients had A1C $>8 \%$. Correlation tests showed that TC reduction was independent of reductions in A1C, WC, and BMI. Reduction of BMI and WC also did not interfere with glycemic control. However, a slight correlation was seen between reduction of TG and BMI $\left(\mathrm{r}^{2}=\right.$ $12.5 \%)$, and TG and $\mathrm{WC}\left(\mathrm{r}^{2}=8 \%\right)$, suggesting a certain degree of co-variance. The correlations calculated can be analyzed by the determination coefficients and $95 \%$ CI shown in Table 3.

None of the files showed reports of severe hypoglycemia, i.e., a situation needing somebody's help. Although there were 16 reports (28\% of the sample) of transient, mild gastrointestinal upset (dyspepsia) at the beginning of metformin use, there was no necessity of dose reduction or withdrawal.

Anti-hypertensive drugs were hydrochlorothiazide, propranolol, and captopril. Patients with dyslipidemia used lovastatin and gemfibrozil. These drugs probably did not interfere with the results, as there was no alteration in dose or type of drug used throughout the study period.

\section{Discussion}

\section{Glycemic control}

The present study showed that the addition of metformin to a scheme of NPH insulin administered twice daily improved glycemic control of type 2 diabetic patients with the metabolic syndrome after 6 months, regardless of BMI reduction, as already shown in other studies $(11,12)$. Although one study showed that metformin can lead to WC reduction (15), the improvement of A1C levels in our sample was independent of WC reduction, indicating that metformin improves sensitivity to the action of insulin by mechanisms already described, such as inhibition of hepatic gluconeogenesis (16). Al- 
though over half the patients reached A1C levels below $8 \%$, only $14 \%$ reached ideal metabolic control (A1C up to 7\%), and $47 \%$ kept their A1C above $8 \%$. More intensive insulin therapy with fast-acting insulins and self-monitoring might have led to better results (17). The mean FBG and A1C values of our patients were similar to those reported in other studies (11-13), although most studies did not report the percentage of patients who reached the established cut-off points for A1C. Such information would have shed light on the extent of glycemic control reached at other centers, since the mean value is sensitive to extreme values.

\section{Lipid profile}

The literature shows discrepant results about the influence of metformin on lipid profile (10). Some studies, in agreement with ours, reported reduction only in TC levels $(18,19)$, while others reported reduction of TC and TG with an increase of HDL-C $(20,21)$. Still other studies showed no changes in lipid profile $(22,23)$. Another investigation showed an association of metformin with an improvement in the lipid profile even in non-diabetic patients (24). New studies are needed to clarify this issue, since TG and HDL-C are very important parameters for the evaluation of metabolic syndrome.

A possible reason for these discrepant results may be that the clinical studies cited above analyzed data from independent samples. In our study, this problem was avoided by paired data analysis, in which each patient was his own control, thereby increasing the power of the statistical analysis.

\section{References}

1. Stratton IM, Adler AI, Neil HA et al. (2000). Association of glycaemia with macrovascular and microvascular complications of type 2 diabetes (UKPDS 35): prospective observational study. British Medical Journal, 321: 405-412.
A recent meta-analysis (10) covering 41 studies on the effects of metformin on BP and lipid profile showed that only TC reduction was significant. Our results are in agreement with these findings and show that this fact was independent of BMI and WC reduction. Since the TG reduction found in the present study underwent interference from BMI and WC (intervening variables), we cannot conclude that metformin had a direct and isolated effect on TG levels.

\section{Other parameters}

The fact that metformin did not reduce BP in our sample agrees with other studies $(10,22,23,25)$, although there are reports of SBP and DBP reduction with this drug $(26,27)$. A possible explanation for this discrepancy may be the fact that not all patients who lose weight experience reductions in their plasma renin and aldosterone levels $(28,29)$. However, metformin is known to positively affect other parameters which influence the development of cardiovascular disease, regardless of any effect on BP (30, 31). Our results agree with the literature regarding the effect of metformin on BMI reduction $(9,12,13)$, reduction of daily insulin dose $(9,12,13)$, biguanide-related sideeffects $(21,24)$, and reduction of WC, which is a cornerstone for the diagnosis of metabolic syndrome and may play the role of a co-variate when other parameters are analyzed $(18,32)$.

In conclusion, our study showed the efficacy of the administration of metformin and insulin simultaneously without negative effects and no changes were detected in HDLcholesterol or blood pressure.
2. Krolewski AS, Laffel LM, Krolewski M et al. (1995). Glycosylated hemoglobin and the risk of microalbuminuria in patients with insulindependent diabetes mellitus. New England Journal of Medicine, 332: 1251-1255. 
3. Turner RC, Cull CA, Frighi V et al. (1999). Glycemic control with diet, sulfonylurea, metformin, or insulin in patients with type 2 diabetes mellitus: progressive requirement for multiple therapies (UKPDS 49). UK Prospective Diabetes Study (UKPDS) Group. Journal of the American Medical Association, 281: 2005-2012.

4. Reaven GM (1988). Banting lecture 1988. Role of insulin resistance in human disease. Diabetes, 37: 1595-1607.

5. Gu K, Cowie CC \& Harris MI (1998). Mortality in adults with and without diabetes in a national cohort of the U.S. population, 19711993. Diabetes Care, 21: 1138-1145.

6. van der Kallen CJ, Voors-Pette C \& de Bruin TW (2004). Abdominal obesity and expression of familial combined hyperlipidemia. Obesity Research, 12: 2054-2061.

7. Lorenzo C, Okoloise M, Williams K et al. (2003). The metabolic syndrome as predictor of type 2 diabetes: the San Antonio heart study. Diabetes Care, 26: 3153-3159.

8. Poirier P, Lemieux I, Mauriege P et al. (2005). Impact of waist circumference on the relationship between blood pressure and insulin: the Quebec Health Survey. Hypertension, 45: 363-367.

9. Despres JP (2003). Potential contribution of metformin to the management of cardiovascular disease risk in patients with abdominal obesity, the metabolic syndrome and type 2 diabetes. Diabetes and Metabolism, 29: 6S53-6S61.

10. Wulffele MG, Kooy A, de Zeeuw D et al. (2004). The effect of metformin on blood pressure, plasma cholesterol and triglycerides in type 2 diabetes mellitus: a systematic review. Journal of Internal Medicine, 256: 1-14.

11. Jaber LA, Nowak SN \& Slaughter RR (2002). Insulin-metformin combination therapy in obese patients with type 2 diabetes. Journal of Clinical Pharmacology, 42: 89-94.

12. Strowig SM, Viles-Santa ML \& Raskin P (2002). Comparison of insulin monotherapy and combination therapy with insulin and metformin or insulin and troglitazone in type 2 diabetes. Diabetes Care, 25: 1691-1698.

13. Wulffele MG, Kooy A, Lehert P et al. (2002). Combination of insulin and metformin in the treatment of type 2 diabetes. Diabetes Care, 25: 2133-2140.

14. Expert Panel on Detection, Evaluation, and Treatment of High Blood Cholesterol in Adults (2001). Executive Summary of the Third Report of the National Cholesterol Education Program (NCEP) Expert Panel on Detection, Evaluation, and Treatment of High Blood Cholesterol in Adults (Adult Treatment Panel III). Journal of the American Medical Association, 285: 2486-2497.

15. Pasquali R, Gambineri A, Biscotti D et al. (2000). Effect of long-term treatment with metformin added to hypocaloric diet on body composition, fat distribution, and androgen and insulin levels in abdominally obese women with and without the polycystic ovary syndrome. Journal of Clinical Endocrinology and Metabolism, 85: 2767-2774.

16. Kirpichnikov D, McFarlane SI \& Sowers JR (2002). Metformin: an update. Annals of Internal Medicine, 137: 25-33.

17. DeWitt DE \& Dugdale DC (2003). Using new insulin strategies in the outpatient treatment of diabetes: clinical applications. Journal of the American Medical Association, 289: 2265-2269.

18. Ginsberg H, Plutzky J \& Sobel BE (1999). A review of metabolic and cardiovascular effects of oral antidiabetic agents: beyond glucoselevel lowering. Journal of Cardiovascular Risk, 6: 337-346.

19. Grant PJ (1996). The effects of high- and medium-dose metformin therapy on cardiovascular risk factors in patients with type II diabetes. Diabetes Care, 19: 64-66.

20. Yki-Jarvinen H, Ryysy L, Nikkila K et al. (1999). Comparison of bedtime insulin regimens in patients with type 2 diabetes mellitus. A randomized, controlled trial. Annals of Internal Medicine, 130: 389396.

21. Robinson AC, Burke J, Robinson S et al. (1998). The effects of metformin on glycemic control and serum lipids in insulin-treated NIDDM patients with suboptimal metabolic control. Diabetes Care, 21: 701-705.

22. Groop L, Widen E, Franssila-Kallunki A et al. (1989). Different effects of insulin and oral antidiabetic agents on glucose and energy metabolism in type 2 (non-insulin-dependent) diabetes mellitus. Diabetologia, 32: 599-605.

23. Rains SG, Wilson GA, Richmond W et al. (1988). The effect of glibenclamide and metformin on serum lipoproteins in type 2 diabetes. Diabetic Medicine, 5: 653-658.

24. DeFronzo RA \& Goodman AM (1995). Efficacy of metformin in patients with non-insulin-dependent diabetes mellitus. The Multicenter Metformin Study Group. New England Journal of Medicine, 333: 541-549.

25. Nagi DK \& Yudkin JS (1993). Effects of metformin on insulin resistance, risk factors for cardiovascular disease, and plasminogen activator inhibitor in NIDDM subjects. A study of two ethnic groups. Diabetes Care, 16: 621-629.

26. Giugliano D, Quatraro A, Consoli G et al. (1993). Metformin for obese, insulin-treated diabetic patients: improvement in glycaemic control and reduction of metabolic risk factors. European Journal of Clinical Pharmacology, 44: 107-112.

27. Landin K, Tengborn L \& Smith U (1991). Treating insulin resistance in hypertension with metformin reduces both blood pressure and metabolic risk factors. Journal of Internal Medicine, 229: 181-187.

28. Manrique C, Lastra G, Whaley-Connell A et al. (2005). Hypertension and the cardiometabolic syndrome. Journal of Clinical Hypertension, 7: 471-476.

29. Tuck ML, Sowers J, Dornfeld L et al. (1981). The effect of weight reduction on blood pressure, plasma renin activity, and plasma aldosterone levels in obese patients. New England Journal of Medicine, 304: 930-933.

30. Katakami N, Yamasaki Y, Hayaishi-Okano R et al. (2004). Metformin or gliclazide, rather than glibenclamide, attenuate progression of carotid intima-media thickness in subjects with type 2 diabetes. Diabetologia, 47: 1906-1913.

31. Schafers RF (2003). Do effects on blood pressure contribute to improved clinical outcomes with metformin? Diabetes and Metabolism, 29: 6S62-6S70.

32. Meenakumari KJ, Agarwal S, Krishna A et al. (2004). Effects of metformin treatment on luteal phase progesterone concentration in polycystic ovary syndrome. Brazilian Journal of Medical and Biological Research, 37: 1637-1644. 